\title{
Helical solutions in scalar gravity
}

\author{
Robert Beig • Bernd G. Schmidt
}

Received: 17 May 2009 / Accepted: 26 May 2009 / Published online: 9 June 2009

(C) The Author(s) 2009. This article is published with open access at Springerlink.com

\begin{abstract}
We construct solutions, for small values of $G$ and angular frequency $\Omega$, of special relativistic scalar gravity coupled to ideally elastic matter which have helical but no stationary or axial symmetry. They correspond to a body without any symmetries in steady rotation around one of its axes of inertia, or two bodies moving on a circle around their center of gravity. Our construction is rigorous, but modulo an unproved conjecture on the differentiability of a certain functional.
\end{abstract}

Keywords Elasticity $\cdot$ Scalar gravity $\cdot$ Helical motion

\section{Introduction}

In Newton's theory of gravity there are solutions describing two bodies which move at constant angular velocity on a circle around their common center of gravity. The existence of such solutions for fluid bodies was demonstrated by Lichtenstein [10]. In Einstein's theory we do not expect such solutions to exist, because such a system will emit gravitational radiation, and so the two bodies will spiral towards each other.

Supported in part by Fonds zur Förderung der Wissenschaftlichen Forschung project no. P20414-N16.

R. Beig $(\varangle)$

Gravitational Physics, Faculty of Physics, University of Vienna,

Boltzmanngasse 5, 1090 Vienna, Austria

e-mail: robert.beig@univie.ac.at

B. G. Schmidt

Max-Planck-Institut für Gravitationsphysik, Albert-Einstein-Institut,

Am Mühlenberg 1, 14476 Golm, Germany

e-mail: bernd@aei.mpg.de 
However, in 1992, Detweiler and Blackburn [8] conjectured that such solutions should exist also in general relativity, provided there is just the right amount of incoming gravitational radiation to keep the bodies on their circle. Friedman, Price and coworkers ([2,9] and references therein), followed this idea and analysed various model systems mathematically and numerically. There are many conceptual and technical problems involved. From the Schild solution [11] of electromagnetism, which describes two point charges moving on a circle, it becomes clear that such a system has infinite energy because the required amount of incoming (and outgoing) radiation is unbounded. So, in GR we expect that the ADM mass will be infinite. Consequently the system will not by asymptotically flat in the sense of any of the known definitions, which in turn makes it difficult to even define such a system. We want the spacetime to admit a Killing vector which behaves like $\partial_{t}+\Omega \partial_{\phi}$. The meaning of this, however, is unclear if there is no asymptotic symmetry group. There are ways out, Friedman proposes a definition in [12].

There is another subtlety: in Minkowski space the helical Killing vector $\partial_{t}+\Omega \partial_{\phi}$ is timelike near the center and spacelike near infinity. Hence, the metric on the quotient of the Killing vector changes signature. It is completely unclear, how to deal with the Einstein equations in such a situation.

All numerical model systems treated so far do not treat the 'bodies' dynamically. In this paper we consider a special relativistic theory in which the bodies are composed of some elastic material and gravity is modeled by a scalar field. The main point of the paper is to demonstrate that solutions do exist in which the incoming radiation keeps the bodies on their circle. In special relativity there is no problem to define such systems. We just want the solution to be invariant under the Killing vector $\partial_{t}+\Omega \partial_{\phi}$. The scalar gravitational field satisfies the wave equation in Minkowski space.

There is a case which is technically simpler than the 2-body system. This is a rigidly rotating 'tri-axial body'. By this we mean that the body does not have to have any symmetries. Again, in Newtons theory such solutions exist [5]. In GR, however, the body should spin down because it radiates gravitational waves if it is not axisymmetric with respect to the axis of the rotation.

The plan of the paper is as follows. In Sect. 2 we describe the theory we are using: special relativistic elasticity formulated as a Lagrangian field theory [3] minimally coupled to a version of scalar gravity. Section 3 discusses helical symmetry and derives the equations for which we want to show existence. In Sect. 4 we consider a triaxial body in helical motion and describe 'its gravitational field' according to scalar gravity. We try to find a solution with the right amount of incoming radiation by taking as gravitational field (1/2 of) the sum of the retarded and advanced solution of a given source motion. With any other combination our method would break down. We want to show existence using the implicit function theorem. Because the linearized operator has a nontrivial range we first solve a 'projected system' first and only then the full system.

In Sect. 5 we discuss in outline the helical 2-body problem for which existence can be established by essentially the same method. We were not able to show that no solution exists if we take just the retarded solution of the wave equation.

There is a gap in our existence proof, because we do not demonstrate that the 'gravitational force' depends differentiably on the configuration, in the function spaces we 
are using for elasticity. We consider this as a technicality. If this point can be cleaned up, we are confident that even for complicated non linear systems such as GR the idea that incoming radiation can balance outgoing radiation works. Finally, we use elastic bodies only because in this case we understand the boundary value problem. A corresponding result should certainly also be true for fluids.

\section{Scalar gravity coupled to elasticity}

Consider a Lagrangian field theory on Minkowski space $M$ space with metric $\eta_{\mu \nu}=$ $\operatorname{diag}(-1,1,1,1)$ with dynamical variables given by a scalar field $V\left(x^{\mu}\right)$ descibing gravity and and the fields $f^{A}\left(x^{\mu}\right)$ describing elasticity in Special Relativity [3], which are viewed as maps $M \rightarrow \mathcal{B}$ with $\mathcal{B}$, the so-called material space or body, being a bounded domain in $\mathbb{R}^{3}$ with smooth boundary $\partial \mathcal{B}$. The action is

$$
S=\frac{1}{2} \int \eta^{\mu \nu} V_{, \mu} V_{, \nu} \sqrt{-\eta} d^{4} x+4 \pi G \int \rho F(V) \sqrt{-\eta} d^{4} x .
$$

We assume that $\mathcal{B}$ is endowed with an Euclidean metric $\delta_{A B}$, where $A, B=1,2,3$ and that the scalar $\rho$ defining the elastic material depends only on the principal invariants of the matrix $H^{A}{ }_{B}=H^{A C} \delta_{B C}$, where $H^{A B}=f^{A}{ }_{, \mu} f^{B}{ }_{, \nu} \eta^{\mu \nu}$. This means, in standard language, that we consider isotropic materials.

The energy momentum tensor of the system is:

$$
T_{\mu \nu}^{\mathrm{tot}}=V_{, \mu} V_{, \nu}-\frac{1}{2} \eta_{\mu \nu} \eta^{\sigma \lambda} V_{, \sigma} V_{, \lambda}+4 \pi G T_{\mu \nu} F
$$

with $T_{\mu \nu}$ the elasticity energy momentum tensor from [3].

$$
T_{\mu \nu}=\rho u_{\mu} u_{v}-\sigma_{\mu \nu}
$$

where $u^{\mu}$ is the future-pointing vector field given by

$$
f_{, \mu}^{A} u^{\mu}=0, \quad \eta_{\mu \nu} u^{\mu} u^{v}=-1
$$

The existence and uniqueness of $u^{\mu}$ is a regularity condition on the fields $f^{A}$ we are considering. The quantity $\sigma_{\mu \nu}$ is given by

$$
-\sigma_{\mu \nu}=n \frac{\partial \epsilon}{\partial H^{A B}} f_{, \mu}^{A} f_{, \nu}^{B},
$$

where

$$
\rho=n \epsilon,
$$

and $n$ is defined by

$$
\varepsilon_{A B C} f_{, \mu}^{A}(x) f^{B}{ }_{, \nu}(x) f_{, \lambda}^{C}(x)=n(x) \epsilon_{\mu \nu \lambda \sigma} u^{\sigma}(x)
$$


The equation for $V$ is

$$
\square V-4 \pi G \rho F^{\prime}=0 .
$$

To obtain a linear equation we choose

$$
F(V)=1+V
$$

We have

$$
\nabla^{v} T_{\mu \nu}^{\mathrm{tot}}=0
$$

The Eqs. $(2.2,2.10)$ imply

$$
0=\rho V_{, \mu}+T_{\mu}{ }^{v} V_{, v}+(1+V)\left(\rho u_{\mu} u^{v}-\sigma_{\mu}{ }^{v}\right)_{, v}
$$

Or $\left(h_{\mu}^{v}=\delta_{\mu}^{v}+u_{\mu} u^{v}\right)$

$$
0=\left[\rho h_{\mu}^{v}-\sigma_{\mu}{ }^{v}\right] V_{, v}+(1+V)\left(\rho u_{\mu} u^{v}-\sigma_{\mu}{ }^{v}\right)_{, v}
$$

Note that in the calculation of $\sigma_{\mu}{ }^{\nu}, \nu$ in terms of the $f^{A}$ the first term in $\rho$ does not contribute. Furthermore, both the 'force term' (involving $V_{v}$ ) in (2.12) and the remaining term is orthogonal to $u^{v}$ (for the latter this is e.g. shown in [3]).

We have a PDE system for $V, f^{A}$. This has a Newtonian limit which is treated in Sect. 5 of [5].

\section{Helical motion}

We assume that the material flow is parallel to the helical Killing vector $\partial_{t}+\Omega \partial_{\phi}$, i.e. that

$$
f_{, \mu}^{A}\left(\partial_{t}+\Omega \partial_{\phi}\right)^{\mu}=0
$$

and that $V$ is invariant under $\partial_{t}+\Omega \partial_{\phi}$, i.e.

$$
V_{\mu}\left(\partial_{t}+\Omega \partial_{\phi}\right)^{\mu}=0
$$

For concreteness we choose coordinates $(\tau, y)$, so that $\tau=0$ coincides with the hyperplane $t=0$ with $y$ Euclidean coordinates thereon and $\partial_{t}+\Omega \partial_{\phi}=\partial_{\tau}$. Explicitly the transformation is given by

$$
x^{1}=y^{1} \cos \Omega \tau-y^{2} \sin \Omega \tau, \quad x^{2}=y^{1} \sin \Omega \tau+y^{2} \cos \Omega \tau, \quad x^{3}=y^{3}, \quad t=\tau
$$


In these coordinates the configuration $f^{A}$ can be written as functions $\hat{f}^{A}$ of $y$, and similarly for $V$. By slight abuse of notation we will omit the bar in what follows. Now Eq. (3.1) implies that the quantities $n, \rho$ and $\sigma_{\mu}{ }^{\nu}$ are all also invariant under $\partial_{t}+\Omega \partial_{\phi}$ and $\sigma_{\mu}^{\nu}\left(\partial_{t}+\Omega \partial_{\phi}\right)^{\mu}=0$. Using the orthogonality pointed out at the end of the previous section and the expression for the projection of $\sigma_{\mu}{ }^{\nu}{ }_{, \nu}$ on the quotient calculated in the paper [5], we obtain

$$
0=\left[\rho h_{j}^{i}-\sigma_{j}^{i}\right] D_{i} V+(1+V)\left[-e^{-U} D_{i}\left(e^{U} \sigma_{j}{ }^{i}\right)+\rho D_{j} U\right],
$$

where $-e^{2 U}$ is the norm of the helical Killing vector given by

$$
e^{2 U}=1-\Omega^{2} r^{2}, \quad r^{2}=\left(x^{1}\right)^{2}+\left(x^{2}\right)^{2}, \quad r^{2} \Omega^{2}<1
$$

$h_{i j}$ is the metric on the quotient of $\partial_{t}+\Omega \partial_{\phi}$ and $D_{i}$ the covariant derivative with respect to $h_{i j}$. Note that, for $\Omega=0, h_{i j}$ equals $\delta_{i j}$. Note also that now $H^{A B}=f^{A}{ }_{, i} f^{B}{ }_{, j} h^{i j}$. We rewrite (3.4) as

$$
D_{i}\left[(1+V) e^{U} \sigma_{j}^{i}\right]-\rho e^{U}\left[D_{j} V+(1+V) D_{j} U\right]=0
$$

The second and third term in (3.6) is respectively the gravitational and centrifugal force. We write (3.6) as

$$
0=D_{i}\left[(1+V) e^{U} \sigma_{j}^{i}\right]+W_{j}+Z_{j}
$$

and

$$
-W_{j}=e^{U} \rho D_{j} V
$$

with

$$
-Z_{j}=e^{U} \rho(1+V) D_{j} U
$$

and

$$
D_{j} U=\Omega^{2}\left(1-\Omega^{2} r^{2}\right)^{-\frac{1}{2}} \frac{1}{2} D_{j} r^{2}
$$

\section{Triaxial rotating body}

The first case we want to consider is a triaxial rotating body coupled to scalar gravity. We repeat that 'tri-axial' means that the set $\mathcal{B}$ need not have any symmetries. Let us denote the corresponding relaxed configuration by $\bar{\Phi}$. We take for the gravitational field the symmetric (i.e. one half the sum of the retarded and advanced) solution of the scalar wave equation. Note that this makes sense globally in Minkowski space. But we 
will only make use of that solution inside the support of the body, where $r^{2} \Omega^{2}<1$. For a source $\rho$ invariant under helical motion we have from the Appendix that

$$
V(y)=G \int H\left(y, y^{\prime}\right) \rho\left(y^{\prime}\right) \sqrt{-\xi^{2}\left(y^{\prime}\right) h\left(y^{\prime}\right)} d y^{\prime},
$$

where $H\left(y, y^{\prime}\right)$ is given by (6.12). Clearly this function is even in $\Omega$. Expanding $V$ in $\Omega$ only even powers occur and we have

$$
V=G \tilde{V}=V^{N}+\Omega^{2} V^{2}+\cdots=G\left(\tilde{V}^{N}+\Omega^{2} \tilde{V}^{2}+\cdots\right)
$$

where $V^{N}$ is the Poisson integral of the source.

\subsection{Solution of the projected system}

The fundamental equation (with $V$ still chosen half the sum of retarded and advanced potential defined by $\rho$ ) is

$$
0=D_{i}\left[(1+V) e^{U} \sigma_{j}^{i}\right]+G \tilde{W}_{j}+\Omega^{2} \tilde{Z}_{j}
$$

with

$$
W_{j}=G \tilde{W}_{j}
$$

and

$$
Z_{j}=\Omega^{2} \tilde{W}_{j}
$$

Equation (4.3) forms a quasilinear second-order system of partial differential equations for the functions $f^{A}$, which has to be solved subject to the (Neumann-type) boundary condition

$$
\left.\sigma_{i}^{j} n_{j}\right|_{f^{-1}(\partial \mathcal{B})}=0
$$

Due to the presence of a free boundary we will also need the material form of (4.3), where the dependent variable $f(y)$ is replaced by its inverse $\Phi(X)$ with $X \in \mathcal{B}$. Then, with $\left.\bar{W}_{j}=n^{-1} \tilde{W}_{j}(\phi(X))\right)$ and $\left.\bar{Z}_{j}=n^{-1} \tilde{Z}_{j}(\phi(X))\right)$, there results

$$
0=F_{j}\left(\Phi, \Omega^{2}, G\right)=\nabla_{A}\left[(1+G \bar{V}) e^{U} \sigma_{j}^{A}\right]+G \bar{W}_{j}+\Omega^{2} \bar{Z}_{j}
$$

where $\sigma^{A}{ }_{j}$ is the 'first Piola stress' given by $\sigma^{A}{ }_{j}=n^{-1} f^{A}{ }_{i} \sigma_{j}{ }_{j}$. The boundary condition now takes the form

$$
\left.\sigma^{A}{ }_{i} n_{A}\right|_{\partial \mathcal{B}}=0
$$

We have two parameters in the problem, namely $\Omega^{2}$ and $G$. 
Now one has to define an appropriate space for $\Phi(X)$, which will be a neighbourhood of the identity map in a suitable Sobolev space, we refer for details to the paper [1]. Let $\bar{\Phi}$ be a relaxed, i.e. stressfree configuration, which we take to be the identity map. We also take $\mathcal{B}$ to be contained in the set $\left[\left(X^{1}\right)^{2}+\left(X^{2}\right)^{2}\right] \Omega^{2}<1$, whence, for deformations $\Phi(X)$ sufficiently close to the identity, the set $\Phi(\mathcal{B})$ is inside the light cylinder $\left[\left(y^{1}\right)^{2}+\left(y^{2}\right)^{2}\right] \Omega^{2}<1$.

We next require that the function $\epsilon\left(H^{A B}\right)$ in (2.6) satisfies

$$
\epsilon\left(\delta^{A B}\right)=\stackrel{\circ}{\epsilon}=\text { const }>0,\left.\quad \frac{\partial \epsilon}{\partial H^{A B}}\right|_{H^{A B}=\delta^{A B}}=0
$$

Then we have a solution $F_{j}(\bar{\Phi}, 0,0)=0$ of (4.7). Assume that furthermore

$$
\left.\left(\frac{\partial^{2} \epsilon}{\partial H^{A B} \partial H^{C D}}\right)\right|_{H^{E F}=\delta^{E F}}=\lambda \delta_{A B} \delta_{C D}+2 \mu \delta_{C(A} \delta_{B) D}
$$

with the constants $\mu, \lambda$ obeying $\mu>0,3 \lambda+2 \mu>0$. The linearization of $F_{j}$ at $(\bar{\Phi}, 0,0)$ is the standard operator of flat-space linear elasticity on $\mathcal{B}$, which has to be considered together with the linearized form of the boundary condition (4.8). It is well-known (see e.g. [1]) that this is an elliptic operator with finite dimensional kernel and range. The latter is given by fields $l_{i}(X)$, such that $\int_{\mathcal{B}} \eta^{A}(X) \delta^{i}{ }_{A} l_{i}(X) d^{3} X=0$ for all Euclidean Killing vectors $\eta^{A}$ on $\mathcal{B}$. One can like in [1] define a projection $\mathbb{P}$ onto the range of the linearized operator and study the projected system

$$
0=\mathbb{P} F_{i}\left(\Phi, \Omega^{2}, G\right)=\mathbb{P}\left\{\nabla_{A}\left[(1+G \bar{V}) e^{\frac{U}{c^{2}}} \sigma_{i}^{A}\right]+G \bar{W}_{i}+\Omega^{2} \bar{Z}_{i}\right\}
$$

Assuming differentiability of this nonlinear map we can apply the implicit function theorem. Differentiability is standard for the $\bar{Z}_{i}$-term (see [5]). For the $\bar{W}_{i}$-term, which contains the retarded + advanced scalar field, we have to leave this as a conjecture. The corresponding result for the Newtonian gravitational field has been proved in [4].

The kernel of the linearized operator consists of Killing vectors of flat Euclidean space. We restrict the deformations $\Phi$ to ones of the form

$$
\Phi^{i}\left(X^{A}, G, \Omega^{2}, a^{i}, b^{j k}\right)=\delta^{i}{ }_{A} X^{A}+a^{i}+b^{i k} \delta_{k A} X^{A}+\tilde{\Phi}
$$

where $a^{i}$ and $b^{i j}$ are constants with $b^{i j}=b^{[i j]}$ and $\tilde{\Phi}$ is in a complement of the kernel. Thus we obtain a unique solution of the projected equations for each value of $a^{i}, b^{i k}$ defining an infinitesimal motion. Hence we know $\tilde{\Phi}\left(X^{A}, G, \Omega^{2}, a^{i}, b^{i k}\right)$. 


\subsection{Equilibration}

To obtain a solution of the full equations we will construct a family $\left(a^{i}\left(G, \Omega^{2}\right), b^{j k}\right.$ $\left.\left(G, \Omega^{2}\right)\right)$ such that

$$
\Phi^{i}\left(X^{A}, G, \Omega^{2}, a^{j}\left(G, \Omega^{2}\right), b^{k l}\left(G, \Omega^{2}\right)\right)
$$

satisfies the equation and the boundary condition. We will determine this family by solving $(\mathbb{I}-\mathbb{P}) F_{i}=0$. This condition is equivalent to

$$
0=\tilde{N}_{(\alpha)}\left(G, \Omega^{2}, C\right)=\int_{\mathcal{B}} \xi_{(\alpha)}^{i}\left\{\nabla_{A}\left[(1+G \bar{V}) e^{U} \sigma_{i}^{A}\right]+G \bar{W}_{i}+\Omega^{2} \bar{Z}_{i}\right\} d^{3} X
$$

for all Killing vectors $\xi_{(\alpha)}^{i}$ with $\alpha=1, \ldots, 6$ of the flat metric on the body, $C=$ $\left(a^{i}, b^{j k}\right)$ and $\Phi^{i}\left(X^{A}, G, \Omega^{2}, C\right)$ is inserted in the integrand. Note that $\xi_{(\alpha)}^{i}=a^{\prime i}+$ $b^{\prime i}{ }_{j} y^{j}$ has to be composed with $\Phi$, so the rotational terms depend on $\Phi^{i}\left(X^{A}, G, \Omega^{2}, C\right)$. To have only one parameter we assume $\omega^{2}=\kappa G$ with $\kappa$ a positive constant and, with this in mind, define the "normalized force map" as

$$
N_{(\alpha)}(G, C)=G^{-1} \int_{\mathcal{B}} \xi_{(\alpha)}^{i}\left\{\nabla_{A}\left[(1+G \bar{V}) e^{U} \sigma^{A}{ }_{i}\right]+G \bar{W}_{i}+\Omega^{2} \bar{Z}_{i}\right\} d^{3} X
$$

and replace (4.14) by

$$
N_{(\alpha)}(G, C)=0
$$

This is well defined because both the forces and $\sigma_{i}^{A}$ have a factor $G$.

In analyzing the 'equilibration condition' (4.16) we show, first of all, that (4.16) is indeed satisfied for arbitrary $G$, when $\xi_{(\alpha)}$ is $\partial_{y^{3}}$ or $y^{2} \partial_{y^{1}}-y^{1} \partial_{y^{2}}$ or a constant linear combination thereof. To prove this, it is simplest to look at the spatial version of (4.16), namely (see (4.3))

$$
G^{-1} \int_{\Phi(\mathcal{B})} \xi_{(\alpha)}^{j}\left\{D_{i}\left[(1+V) e^{U} \sigma_{j}^{i}\right]+G \tilde{W}_{j}+\Omega^{2} \tilde{Z}_{j}\right\} \sqrt{h} d^{3} y=0
$$

Now, the first term in (4.17) is zero: use integration by parts, the Killing equation for each of these two vectors and the vanishing of the boundary term by virtue of (4.6). For the third contribution already the integrand is zero, again by the symmetries of $U$. Finally, the second term is zero: this amounts to the statement, proven in the Appendix, that, for a body which rotates rigidly around the $y^{3}$-axis, the $y^{3}$-component of both the force and torque due to its own gravitational field is zero, provided this field is given by the symmetric solution of the wave Eq. (2.8). 
We thus only consider

$$
N_{\left(\alpha^{\prime}\right)}\left(G, C^{\prime}\right)=0
$$

where $C^{\prime}=\left(a^{1}, a^{2}, a^{3}=0, b^{12}=0, b^{13}, b^{23}\right)$ and $\xi_{\left(\alpha^{\prime}\right)}$ to be the collection

$$
\left\{\xi_{(1)}, \xi_{(2)}, \xi_{(13)}, \xi_{(23)}\right\}=\left\{\partial_{x^{1}}, \partial_{y^{2}}, y^{3} \partial_{y^{1}}-y^{1} \partial_{y^{3}}, y^{3} \partial_{y^{2}}-y^{2} \partial_{y^{3}}\right\}
$$

Suppose $N_{\left(\alpha^{\prime}\right)}(0,0)=0$ and $\frac{\partial N_{\left(\alpha^{\prime}\right)}}{\partial C^{\prime}}(0,0)$ is invertible. Then, by the (finite-dimensional) implicit function theorem, for sufficiently small $G$ there exists a function $C^{\prime}(G)$ such that $N_{\left(\alpha^{\prime}\right)}\left(G, C^{\prime}(G)\right)=0$, and we have a solution of our problem.

Calculating $N(0,0)$ is the same as $\frac{\partial \tilde{N}}{\partial G}$. The first term in the integrand gives $\partial_{A} \delta \sigma_{i}^{A}$ which is equilibrated as was discussed in Eq. (4.2) of [1]. So we have only to consider the force terms.

$$
N_{\left(\alpha^{\prime}\right)}\left(G, C^{\prime}\right)=\int_{\mathcal{B}} \xi_{\left(\alpha^{\prime}\right)}^{i}\left(\bar{W}_{i}+\kappa \bar{Z}_{i}\right) d^{3} X
$$

We obtain

$$
N_{\left(\alpha^{\prime}\right)}\left(0, C^{\prime}\right)=-\int_{\mathcal{B}}\left[\xi_{\left(\alpha^{\prime}\right)}^{1}(\Psi) \Psi^{1}+\xi_{\left(\alpha^{\prime}\right)}^{2}(\Psi) \Psi^{2}\right] d^{3} X+\int_{\mathcal{B}} \xi_{\left(\alpha^{\prime}\right)}^{i}(\Psi) \bar{W}_{i}^{N}(\Psi) d^{3} X
$$

where $\Psi=\delta_{A}^{i} X^{A}+a^{i}+b^{i k} \delta_{k A} X^{A}$ with $a^{3}=b^{12}=0$. The $\bar{W}_{i}^{N}$-term is simply the Newtonian gravitational self-force (resp. self-torque), and so its contribution to the integral vanishes (see e.g. example (i) in the Appendix). We are thus left with

$$
N_{\left(\alpha^{\prime}\right)}\left(0, C^{\prime}\right)=-\int_{\mathcal{B}}\left[\xi_{\left(\alpha^{\prime}\right)}^{1}(\Psi) \Psi^{1}+\xi_{\left(\alpha^{\prime}\right)}^{2}(\Psi) \Psi^{2}\right] d^{3} X
$$

where

$$
\begin{aligned}
& N_{(1)}\left(0, C^{\prime}\right)=-\int_{\mathcal{B}}\left(X^{1}+a^{1}+b^{13} X^{3}\right) d^{3} X \\
& N_{(2)}\left(0, C^{\prime}\right)=-\int_{\mathcal{B}}\left(X^{2}+a^{2}+b^{23} X^{3}\right) d^{3} X \\
& N_{(13)}\left(0, C^{\prime}\right)=-\int_{\mathcal{B}} X^{3}\left(X^{1}+a^{1}+b^{13} X^{3}\right) d^{3} X \\
& N_{(23)}\left(0, C^{\prime}\right)=-\int_{\mathcal{B}} X^{3}\left(X^{2}+a^{2}+b^{23} X^{3}\right) d^{3} X
\end{aligned}
$$


From here we infer that $N_{\left(\alpha^{\prime}\right)}(0,0)$ is zero iff (i) the rotation axis in the reference estate goes through the center of mass and (ii) coincides with one of the axes of inertia. In fact, we assume that the center of mass is at the origin, rather than just (i). With these assumptions minus the matrix $\frac{\partial N_{\left(\alpha^{\prime}\right)}}{\partial C^{\prime}}(0,0)$ is given by

$$
\left(\begin{array}{llll}
\mathcal{V} & 0 & 0 & 0 \\
0 & \mathcal{V} & 0 & 0 \\
0 & 0 & \mathcal{W} & 0 \\
0 & 0 & 0 & \mathcal{W}
\end{array}\right)
$$

where

$$
\mathcal{V}=\int_{\mathcal{B}} d^{3} X \quad \mathcal{W}=\int_{\mathcal{B}}\left(X^{3}\right)^{2} d^{3} X
$$

So $\frac{\partial N_{\left(\alpha^{\prime}\right)}}{\partial C^{\prime}}(0,0)$ is invertible, and our argument is complete.

\section{Two bodies in circular motion}

In this section we consider two identical bodies in circular motion around their common center. To simplify matters we assume that the support of the matter in the relaxed configuration consists of two spherical balls with centers at $(-L, 0,0)$ and $(L, 0,0)$ on the quotient metric. We have as discrete symmetry the reflections $\left(y^{1}, y^{2}, y^{3}\right) \rightarrow$ $\left(y^{1},-y^{2}, y^{3}\right)$ and $\left(y^{1}, y^{2}, y^{3}\right) \rightarrow\left(y^{1}, y^{2},-y^{3}\right)$. For an isotropic stored energy functions we know that the solution will also have this symmetry. Therefore we consider only configurations with the property

$$
\begin{aligned}
& \Phi^{1}\left(X^{1}, X^{2}, X^{3}\right)=\Phi^{1}\left(X^{1},-X^{2}, X^{3}\right)=\Phi^{1}\left(X^{1}, X^{2},-X^{3}\right) \\
& \Phi^{1}\left(X^{1}, X^{2}, X^{3}\right)=-\Phi^{1}\left(X^{1},-X^{2}, X^{3}\right)=\Phi^{1}\left(X^{1}, X^{2},-X^{3}\right) \\
& \Phi^{1}\left(X^{1}, X^{2}, X^{3}\right)=\Phi^{1}\left(X^{1},-X^{2}, X^{3}\right)=-\Phi^{1}\left(X^{1}, X^{2},-X^{3}\right)
\end{aligned}
$$

One is now able to show the following:

1. For such configurations the gravitational field "ad+ret" inherits this symmetry. So does the centrifugal force.

2. For an isotropic stored energy also the stress tensor and its divergence inherit this symmetry because it is an isometry of the quotient metric.

3. All these properties imply that all equilibration integrals, with the exception of that for the $y^{1}$-translation Killing vector, are satisfied for any configuration with these symmetries.

4. If we assume further that $y^{1} \rightarrow-y^{1}$, which exchanges the two bodies, is a symmetry we have only to consider the elastic equations for one body.

5. As usual, we first solve the projected equations with a parameter $a^{1}$, the translation in the $y^{1}$-direction, and have then to study the bifurcation equation $N\left(G, a^{1}\right)=0$. 
It turns out that $N\left(0, a^{1}\right)=0$ is the same as in the Newtonian problem which we solved in [6]. We find a reference configuration with $N(0,0)=0$ and $\frac{\partial N}{\partial a}(0,0)=$ 0 and have thus solved the 2-body problem.

Acknowledgments Jürgen Ehlers has greatly influenced our scientific thinking. We dedicate this work to his memory.

Open Access This article is distributed under the terms of the Creative Commons Attribution Noncommercial License which permits any noncommercial use, distribution, and reproduction in any medium, provided the original author(s) and source are credited.

\section{Appendix}

In this Appendix we prove a result which contains as a special case the statement that the gravitational contribution to the equilibration integrals (4.15) vanishes, when the Killing vector is taken to be $\partial_{x^{3}}$ or $\partial_{\phi}=-x^{2} \partial_{x^{1}}+x^{1} \partial_{x^{2}}$.

Let $\rho(t, x)$ be a source and

$$
V(t, x)=\int G\left(t-t^{\prime} ; x-x^{\prime}\right) \rho\left(t^{\prime}, x^{\prime}\right) d t^{\prime} d^{3} x^{\prime}
$$

be the field, where $G$ is 'some unique' Green function (in fact: distribution) of $\square \Phi=\rho$. By 'some unique Green function' we mean a Green function sharing the symmetry of the background (i.e. Minkowski space in our case), such as the retarded or advanced Green function. We assume that $\rho$ has compact support in space.

Let us next suppose that the source $\rho$ is invariant under the flow of some timelike Killing vector $\xi^{\mu} \partial_{\mu}$. Using coordinates comoving with $\xi$, i.e. $(\tau ; y)$ so that $\xi^{\mu} \partial_{\mu}=\partial_{\tau}$, Eq. (1) takes the form

$$
V(\tau, y)=\int H\left(y, y^{\prime}\right) \rho\left(y^{\prime}\right) \sqrt{-\xi^{2}\left(y^{\prime}\right) h\left(y^{\prime}\right)} d^{3} y^{\prime},
$$

where $H\left(y, y^{\prime}\right)=\int G\left(\tau-\tau^{\prime} ; y, y^{\prime}\right) d \tau^{\prime}, \xi^{2}=g_{\mu \nu} \xi^{\mu} \xi^{\nu}$ and $h$ is the determinant of the metric on the quotient of $\xi^{\mu}$ (the square root in (2) is $\sqrt{-g}$ in the comoving system). Clearly $V$ is actually independent of $\tau$.

Let now $\eta$ be a spacelike Killing field commuting with $\xi$, i.e. projecting to $\eta=$ $\eta^{i}(y) \partial_{i}$ on the quotient. Consider the expression $F_{\eta}$ given by

$$
F_{\eta}=\int \rho(y)\left[\eta^{i} \partial_{i} H\left(y, y^{\prime}\right)\right] \rho\left(y^{\prime}\right) \sqrt{-\xi^{2}(y) h(y)} \sqrt{-\xi^{2}\left(y^{\prime}\right) h\left(y^{\prime}\right)} d^{3} y d^{3} y^{\prime}
$$

This is up to sign the equilibration integral $\int_{f^{-1}(\mathcal{B})} \eta^{i} W_{i} \sqrt{h} d^{3} y$. Since the (unique) Green function shares the symmetry of the background there has to hold (operate on both arguments with the symmetry and linearize)

$$
\left[\eta^{i}(y) \partial_{i}+\eta^{i^{\prime}}\left(y^{\prime}\right) \partial_{i^{\prime}}\right] H\left(y, y^{\prime}\right)=0
$$


(Note this relation holds irrespective of whether we take the retarded, advanced or any other combination.) Furthermore $\eta^{i} \partial_{i}$ is a Killing vector in the quotient space, so that in particular

$$
\partial_{i}\left(\sqrt{h} \eta^{i}\right)=0
$$

Also there holds

$$
\eta^{i} \partial_{i} \xi^{2}=0
$$

We use $(6.4,6.5,6.6)$ and integration by parts in $y^{\prime}$ to obtain (the two minus-signs arising in the process cancel)

$$
F_{\eta}=\int \rho(y) H\left(y, y^{\prime}\right)\left[\eta^{i^{\prime}}\left(y^{\prime}\right) \partial_{i^{\prime}} \rho\left(y^{\prime}\right)\right] \sqrt{-\xi^{2}(y) h(y)} \sqrt{-\xi^{2}\left(y^{\prime}\right) h\left(y^{\prime}\right)} d^{3} y d^{3} y^{\prime}
$$

But we can also perform partial integration in the $y$-variable to find that

$$
F_{\eta}=-\int\left[\eta^{i}(y) \partial_{i} \rho(y)\right] H\left(y, y^{\prime}\right) \rho\left(y^{\prime}\right) \sqrt{-\xi^{2}(y) h(y)} \sqrt{-\xi^{2}\left(y^{\prime}\right) h\left(y^{\prime}\right)} d^{3} y d^{3} y^{\prime}
$$

We now assume that the Green function is the symmetric one, so that $G$ is invariant under the joint interchange of $\left(\tau, \tau^{\prime}\right)$ and $\left(y, y^{\prime}\right)$. It follows that $H\left(y, y^{\prime}\right)=H\left(y^{\prime}, y\right)$. Whence, by $(6.3,6.8)$, we find that $F_{\eta}$ is zero. We illustrate the previous result by two examples:

(i) Static symmetry: Here $\xi$ is given by $\xi^{\mu} \partial_{\mu}=\partial_{t}$, and the, say retarded, Green function in adapted coordinates takes the well-known form $(\tau=t, y=x)$ :

$$
4 \pi G_{\text {ret }}\left(\tau-\tau^{\prime} ; y, y^{\prime}\right)=\frac{\delta\left(\tau-\tau^{\prime}-\left|y-y^{\prime}\right|\right)}{\left|y-y^{\prime}\right|}
$$

Furthermore

$$
4 \pi H\left(y, y^{\prime}\right)=\frac{1}{\left|y-y^{\prime}\right|}
$$

i.e. $H\left(y, y^{\prime}\right)$ is the Poisson kernel (this result is of course independent on which combination of the retarded and advanced Green function has been chosen). The quotient metric $h$ is the Euclidean one and spacetime Killing vectors projecting to the quotient in this case are simply all Euclidean ones in $y$-space. We have thus recovered the well-known Newtonian result, that the force and torque on a static body due to its own gravitational field are zero.

(ii) Helical symmetry (see [7]): Here $\xi$ is given by $\xi^{\mu} \partial_{\mu}=\partial_{t}+\Omega \partial_{\phi}$, and the retarded Green function takes the form $\left(\tau=t, \mu=\phi-\Omega t, z=x^{3}\right)$ 


$$
\begin{aligned}
& 4 \pi G_{\mathrm{ret}}\left(\tau-\tau^{\prime} ; y, y^{\prime}\right) \\
& =\frac{\delta\left(\tau-\tau^{\prime}-\sqrt{r^{2}+r^{\prime 2}-2 r r^{\prime} \cos \left[\mu-\mu^{\prime}+\Omega\left(\tau-\tau^{\prime}\right)\right]+\left(z-z^{\prime}\right)^{2}}\right)}{\sqrt{r^{2}+r^{\prime 2}-2 r r^{\prime} \cos \left[\mu-\mu^{\prime}+\Omega\left(\tau-\tau^{\prime}\right)\right]+\left(z-z^{\prime}\right)^{2}}}
\end{aligned}
$$

whence (assuming $r^{2} \Omega^{2}<1$ )

$$
4 \pi H\left(y, y^{\prime}\right)=\frac{1}{2}\left(\frac{1}{\sigma_{+}\left(\mu-\mu^{\prime}, r, r^{\prime}, z-z^{\prime}\right)}-\frac{1}{\sigma_{-}\left(\mu-\mu^{\prime}, r, r^{\prime}, z-z^{\prime}\right)}\right),
$$

with $\sigma_{ \pm}\left(\mu, r, r^{\prime}, z\right)$ being implicitly given by

$$
\sigma_{ \pm}= \pm \sqrt{r^{2}+r^{\prime 2}-2 r r^{\prime} \cos \left(\mu+\Omega \sigma_{ \pm}\right)+z^{2}}
$$

(If we had not chosen the symmetric Green function the quantity corresponding to $H\left(y, y^{\prime}\right)$ would have been different, in particular not symmetric.) The quotient metric $h$ is given by

$$
h_{i j} d x^{i} d x^{j}=d r^{2}+\frac{r^{2}}{1-\Omega^{2} r^{2}} d \mu^{2}+d z^{2}
$$

and spacetime Killing vectors projecting to the quotient are given by $\partial_{x^{3}}=\partial_{z}$ and $\partial_{\phi}=\partial_{\mu}$. Applying the main statement of this Appendix to these two Killing vectors yields the result concerning the second term in Eq. (4.17).

\section{References}

1. Andersson, L., Beig, R., Schmidt, B.G.: Static self-gravitating elastic bodies in Einstein gravity. Commun. Pure Appl. Math. 61, 988-1023 (2008)

2. Beetles, C., Bromley, B., Price, R.H.: The periodic standing-wave approximation: Eigenspectral computations for linear gravity and nonlinear toy models. Phys. Rev. D 74, 024013 (2006)

3. Beig, R., Schmidt, B.G.: Relativistic elasticity. Class. Quantum Gravity 20, 889-904 (2003)

4. Beig, R., Schmidt, B.G.: Static, self-gravitating elastic bodies. Proc. R. Soc. Lond. A 459, 109-115 (2003)

5. Beig, R., Schmidt, B.G.: Relativistic elastostatics I: Bodies in rigid rotation. Class. Quantum Gravity 22, 2249-2268 (2005)

6. Beig, R., Schmidt, B.G.: Celestial mechanics of elastic bodies. Math. Z. 258, 381-394 (2008)

7. Beig, R., Heinzle, J.M., Schmidt, B.G.: Helically symmetric N-particle solutions in scalar gravity. Phys. Rev. Lett. 98, 121102 (2007)

8. Detweiler, S., Blackburn, J.K.: Close black-hole binary systems. Phys. Rev. D 46, 2318-2333 (1992)

9. Friedman, J.L., Uryu, K.: Thermodynamics of binary black holes and neutron stars. Phys. Rev. D 65, 064035 (2002)

10. Lichtenstein, L.: Untersuchungen über die Gestalt der Himmelskörper. Math. Z. 12, 201-218 (1922)

11. Schild, A.: Electromagnetic two-body problem. Phys. Rev. 131, 2762-2766 (1963)

12. Yoshida, S., Bromley, B.C., Read, K., Uryu, K., Friedman, J.L.: Models of helically symmetric binary systems. Class. Quantum Gravity 23, S599-S614 (2006) 